\title{
Artificial neural network retrained to detect myocardial ischemia using a Japanese multicenter database
}

\author{
Kenichi Nakajima $^{1}$ (D) Koichi Okuda ${ }^{2} \cdot$ Satoru Watanabe $^{1} \cdot$ Shinro Matsuo $^{1} \cdot$ Seigo Kinuya $^{1} \cdot$ Karin Toth $^{3} \cdot$ \\ Lars Edenbrandt ${ }^{4}$
}

Received: 12 February 2018 / Accepted: 4 March 2018 / Published online: 7 March 2018

(c) The Author(s) 2018. This article is an open access publication

\begin{abstract}
Purpose An artificial neural network (ANN) has been applied to detect myocardial perfusion defects and ischemia. The present study compares the diagnostic accuracy of a more recent ANN version (1.1) with the initial version 1.0.

Methods We examined 106 patients (age, $77 \pm 10$ years) with coronary angiographic findings, comprising multi-vessel disease ( $\geq 50 \%$ stenosis) (52\%) or old myocardial infarction (27\%), or who had undergone coronary revascularization (30\%). The ANN versions 1.0 and 1.1 were trained in Sweden $(n=1051)$ and Japan $(n=1001)$, respectively, using ${ }^{99 \mathrm{~m}} \mathrm{Tc}-\mathrm{methoxy}$ isobutylisonitrile myocardial perfusion images. The ANN probabilities (from 0.0 to 1.0) of stress defects and ischemia were calculated in candidate regions of abnormalities. The diagnostic accuracy was compared using receiver-operating characteristics (ROC) analysis and the calculated area under the ROC curve (AUC) using expert interpretation as the gold standard. Results Although the AUC for stress defects was 0.95 and $0.93(p=0.27)$ for versions 1.1 and 1.0, respectively, that for detecting ischemia was significantly improved in version $1.1(p=0.0055)$ : AUC 0.96 for version 1.1 (sensitivity 87\%, specificity 96\%) vs. 0.89 for version 1.0 (sensitivity 78\%, specificity $97 \%$ ). The improvement in the AUC shown by version 1.1 was also significant for patients with neither coronary revascularization nor old myocardial infarction $(p=0.0093)$ : AUC $=0.98$ for version 1.1 (sensitivity $88 \%$, specificity $100 \%$ ) and 0.88 for version 1.0 (sensitivity $76 \%$, specificity $100 \%$ ). Intermediate ANN probability between 0.1 and 0.7 was more often calculated by version 1.1 compared with version 1.0, which contributed to the improved diagnostic accuracy. The diagnostic accuracy of the new version was also improved in patients with either single-vessel disease or no stenosis $(n=47$; AUC, 0.81 vs. 0.66 vs. $p=0.0060)$ when coronary stenosis was used as a gold standard.
\end{abstract}

Conclusion The diagnostic ability of the ANN version 1.1 was improved by retraining using the Japanese database, particularly for identifying ischemia.

Keywords Nuclear cardiology $\cdot$ Artificial intelligence $\cdot$ Myocardial perfusion imaging $\cdot$ Coronary artery disease

\section{Abbreviations}

ANN Artificial neural network

AUC Area under the curve

MIBI Methoxyisobutylisonitrile

Kenichi Nakajima

nakajima@med.kanazawa-u.ac.jp

1 Department of Nuclear Medicine, Kanazawa University Hospital, 13-1 Takara-machi, Kanazawa 920-8641, Japan

2 Department of Physics, Kanazawa Medical University, Uchinada, Kahoku, Japan

3 EXINI Diagnostics, Lund, Sweden

4 Department of Clinical Physiology and Nuclear Medicine, University of Gothenburg, Gothenburg, Sweden
MPI Myocardial perfusion imaging

OMI Old myocardial infarction

ROC Receiver-operating characteristics

SDS Summed difference score

SRS Summed rest score

SSS Summed stress score

\section{Introduction}

The diagnostic ability of artificial neural network (ANN), which is a type of artificial intelligence, has been examined from the viewpoint of nuclear cardiology applications [1, 2]. A multicenter study was the first in Japan to apply an ANN to myocardial perfusion imaging (MPI) during 2015 
[3]. That ANN was trained to detect myocardial stress perfusion defects and induced ischemia on a Swedish database, but its diagnostic ability was comparable to that of expert interpretation for Japanese patients. Thereafter, the diagnostic ability was further improved by training the ANN on a Japanese multicenter database $(n=1,001)$ using ${ }^{99 \mathrm{~m}} \mathrm{Tc}$ methoxyisobutylisonitrile (MIBI) MPI [4]. That validation study indicated that the ANN had good diagnostic ability comparable to nuclear cardiology expert interpretation, as the area under the receiver-operating characteristics (ROC) curve (AUC) was 0.92 .

However, whether or not the diagnostic accuracy of version 1.1 actually improved from the initial cardioREPO software version 1.0 (FUJIFILM RI Pharma Co. Ltd., EXINI Diagnostics, Lund, Sweden) has not been validated. In addition, the conditions under which the diagnostic ability of version 1.1 changed have remained unknown. The present study aimed to determine whether the diagnostic ability of version 1.1 trained on a Japanese database was improved over the original version by comparison with the same population that was used before [3].

\section{Methods}

\section{Participants}

The participants were as described for the validation study of the first version (cardioREPO version 1.0) [3]. A total of 106 patients (male, $61 \%$; mean age, $70 \pm 10$ years) who underwent coronary angiography within 1 month of MPI were selected from Public Central Hospital of Matto Ishikawa, Kanazawa Cardiovascular Hospital, and Kanazawa University Hospital. When the number of vessels with coronary stenosis $\geq 50 \%$ was defined as abnormal, 25,29 , 30 and 22 patients had 0,1-, 2-, and 3-vessel disease (total of patients with multi-vessel disease: $52 \%$ ). Comorbidities comprised hypertension (58\%), diabetes mellitus (33\%) and dyslipidemia (36\%), and 27 and $30 \%$ of the patients had a clinical history of old myocardial infarction and coronary revascularization, respectively. All clinical data were completely anonymized and processed at Kanazawa University. The Ethics Committee at Kanazawa University approved the study.

\section{Myocardial perfusion imaging and diagnosis}

Patients were assessed using a stress-rest sequence with a standard dose (maximum, 1,110 MBq) and a protocol for ${ }^{99 \mathrm{~m}} \mathrm{Tc}$ perfusion tracers [5]. Acquired energy was centered at a ${ }^{99 \mathrm{~m}} \mathrm{Tc}$ window of $140 \mathrm{keV} \pm 10 \%$. Stress was imposed either by exercise $(89 \%)$ or pharmacologically using adenosine (11\%). Electrocardiographic gating on the dualheaded SPECT system was 16 frames per cardiac cycle. Attenuation and scatter correction were not applied.

Left ventricular ejection fraction and volumes were also calculated $[6,7]$.

The final diagnoses of ischemia or infarction were the same as those in the first report [3]. Briefly, a diagnosis was concluded based on the consensus of three experienced nuclear medicine physicians similar to clinical diagnostic procedures to determine ischemia. Original short-axis images and polar maps were presented with information only about age and sex. Left ventricular function, including volumes and ejection fraction, was then added, and all subsequent information about coronary artery stenosis, restenosis, and location of stents or bypass grafts was added. Therefore, the presence of a stress abnormality and of stress-induced ischemia was determined based on the integrated understanding of coronary stenosis and the presence of infarction.

\section{Artificial neural network training}

The first version of the ANN was trained on data from 1,051 Swedish patients (male: $47 \%$; age, $62 \pm 10$ years) and experienced Swedish physicians classified perfusion as normal or defective [2]. Twelve hospitals in Japan collaborated to train version 1.1 ( $n=1,001$ patients; $75 \%$ male; $69 \pm 10$ years) using ${ }^{99 \mathrm{~m}} \mathrm{Tc}$-MIBI as the tracer [4]. At least two Japanese nuclear cardiology experts determined abnormal stress defects and stress-induced ischemia by consensus. Areas of possible perfusion abnormalities in stress and rest images (stress and rest defects, respectively) were segmented, and the ANN judged candidate regions in terms of the probability of abnormalities (ANN probability) based on 16 features extracted from the shape, extent, location, count, perfusion homogeneity, regional motion, wall thickening and sex.

\section{Defect scoring}

Scoring was based on a 17-segment model [8] and a 5-point scale $(0$, normal; 1 , slight decrease; 2 , moderate decrease; 3 , severe decrease; 4 , complete defect) and calculated automatically by the cardioREPO software (version 1.1). Summed stress (SSS), summed rest (SRS) and summed difference (SDS) scores were included. Defect severity was classified using the database of the Japanese Society of Nuclear Medicine working group that included normal stress-rest findings on SPECT images that were acquired using an Anger camera and not attenuation-corrected $[9,10]$.

\section{Statistics}

Data are shown as means \pm standard deviation (SD). Differences between groups were assessed using a one-way 
analysis of variance, Student's $T$ tests and $F$ tests, and areas under ROC curves were calculated using JMP version 12 (SAS Institute Inc., Cary, NC, USA) statistics software. The appropriate threshold values for sensitivity and specificity were determined at the point at which the maximum sensitivity + specificity -1 was obtained. A significant difference was indicated when $p<0.05$.

\section{Results}

Figure 1 shows differences in the segmentation of abnormal regions between versions 1.0 and 1.1 in a patient with anterior myocardial infarction accompanied by exercise-induced ischemia. The area of ischemia was small (probability, 0.96; extent, $3 \%$ ) in version 1.0. A larger area with a probability of 0.88 and an extent of $9 \%$ was identified in the anterior wall, but a small basal region that was selected as candidate was determined as insignificant (probability, < 0.5 ).

Stress defects and induced ischemia were compared between ANN probability determined by both software versions and expert interpretation (Table 1). The ANN probability values for patients with and without stress defects were $0.87 \pm 0.21$ and $0.25 \pm 0.34$, respectively $(F$ ratio, $134 ; p<0.0001)$, with version 1.0 , and $0.85 \pm 0.21$ and $0.23 \pm 0.28$, respectively, with version 1.1 ( $F$ ratio $170 ; p<0.0001)$. Values for patients with and without stress-induced ischemia were $0.70 \pm 0.40$ and $0.01 \pm 0.10$ $(F$ ratio $152 ; p<0.0001)$ with version 1.0 , and $0.79 \pm 0.20$ and $0.21 \pm 0.22$ with version 1.1 ( $F$ ratio, $195 ; p<0.0001)$, respectively.

Figures 2 and 3 show the results of ROC analyses for detecting stress defects and induced ischemia, respectively, and statistical measures of sensitivity, specificity, and accuracy. Figure 2 shows that the AUC for detecting stress defects calculated by versions 1.0 and 1.1 were 0.93 and 0.95 , respectively, which did not significantly differ $(p=0.27)$. The AUC did not significantly differ $(p=0.49$ and 1.00) even when patients were divided into groups without either revascularization or old myocardial infarction (OMI), and with revascularization and/or OMI. In contrast, Fig. 3 shows that the AUC for ischemia was better for version 1.1 (0.96) than for version $1.0(0.89$, $p=0.0055)$. The AUC was better for version $1.1(0.98)$ than for version $1.0(0.88, p=0.0093)$ when patients had neither revascularization nor OMI, but did not differ significantly between those with revascularization and/ or OMI $(p=0.42)$. Using the version 1.1 sensitivity and

\section{A Version 1.0}
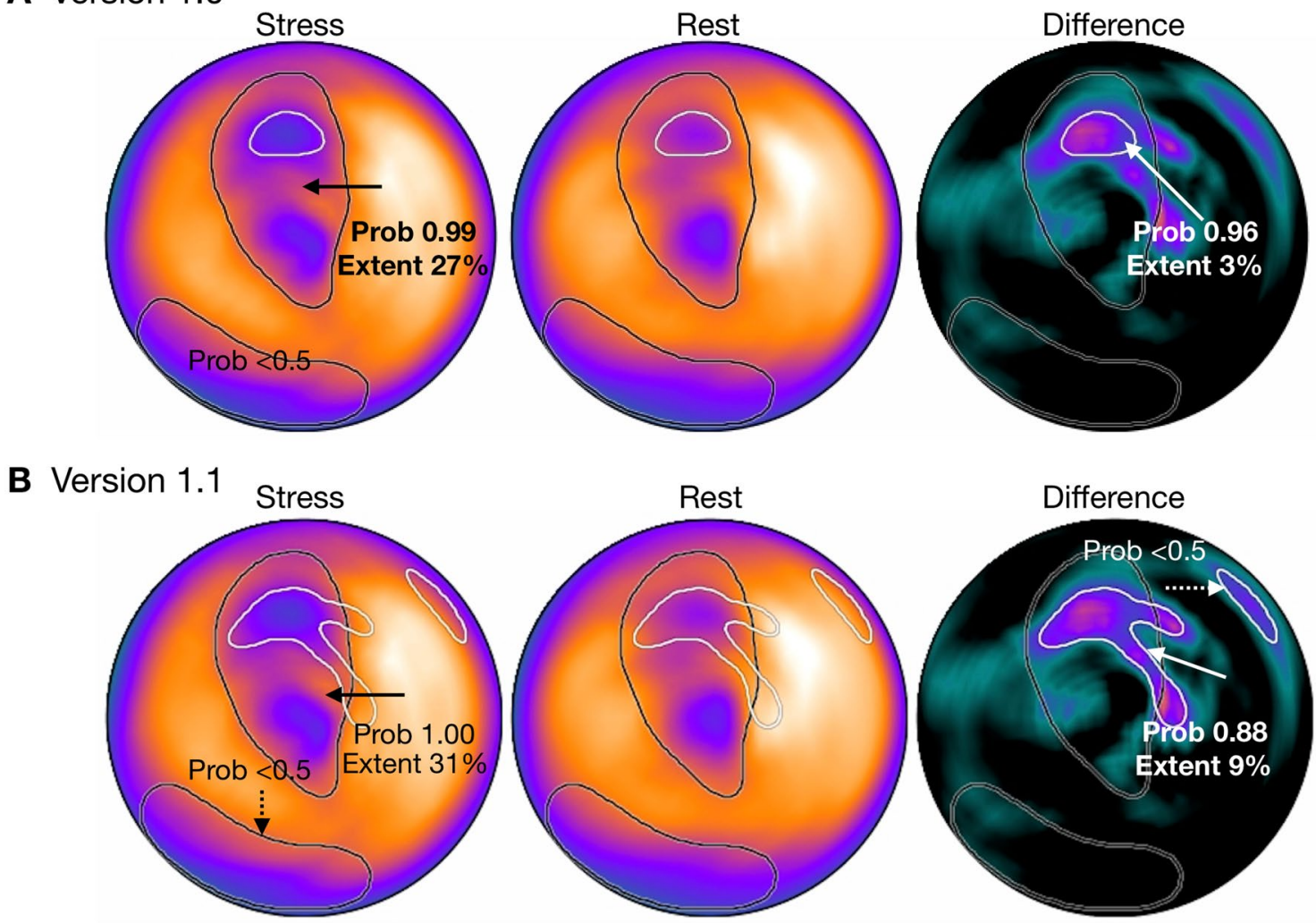

Fig. 1 Comparison of segments of abnormality between versions 1.0 and 1.1. ANN probability (Prob) and extent (\%) are shown as stress and difference polar maps 
Table 1 ANN probabilities and expert interpretation by versions 1.0 and 1.1

\begin{tabular}{|c|c|c|c|c|c|c|c|}
\hline & $N$ & Mean & SD & $\begin{array}{l}\text { Lower } 95 \% \\
\text { of mean }\end{array}$ & $\begin{array}{l}\text { Upper } 95 \% \text { of } \\
\text { mean }\end{array}$ & $F$ ratio & $p$ \\
\hline \multicolumn{8}{|l|}{ A. Stress defect } \\
\hline \multicolumn{8}{|l|}{ Version 1.0} \\
\hline No stress defect & 37 & 0.25 & 0.34 & 0.13 & 0.36 & \multirow[t]{2}{*}{134} & \multirow[t]{2}{*}{$<0.0001$} \\
\hline Stress defect & 69 & 0.87 & 0.21 & 0.82 & 0.92 & & \\
\hline \multicolumn{8}{|l|}{ Version 1.1} \\
\hline No stress defect & 37 & 0.23 & 0.28 & 0.14 & 0.32 & \multirow[t]{2}{*}{170} & \multirow[t]{2}{*}{$<0.0001$} \\
\hline Stress defect & 69 & 0.85 & 0.21 & 0.80 & 0.91 & & \\
\hline \multicolumn{8}{|l|}{ B. Induced ischemia } \\
\hline \multicolumn{8}{|l|}{ Version 1.0} \\
\hline No ischemia & 55 & 0.01 & 0.10 & -0.01 & 0.04 & \multirow[t]{2}{*}{152} & \multirow[t]{2}{*}{$<0.0001$} \\
\hline Ischemia & 51 & 0.70 & 0.40 & 0.59 & 0.82 & & \\
\hline \multicolumn{8}{|l|}{ Version 1.1} \\
\hline No ischemia & 55 & 0.21 & 0.22 & 0.15 & 0.27 & \multirow[t]{2}{*}{195} & \multirow[t]{2}{*}{$<0.0001$} \\
\hline Ischemia & 51 & 0.79 & 0.20 & 0.73 & 0.85 & & \\
\hline
\end{tabular}

$A N N$ artificial neural network, $S D$ standard deviation
A All patients

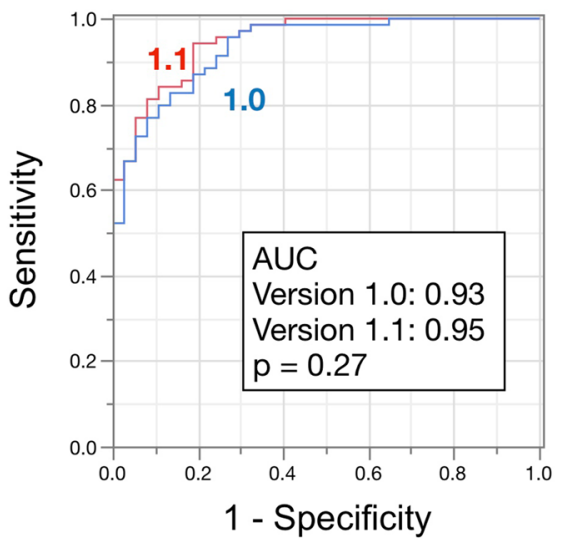

B Neither revascularization nor OMI

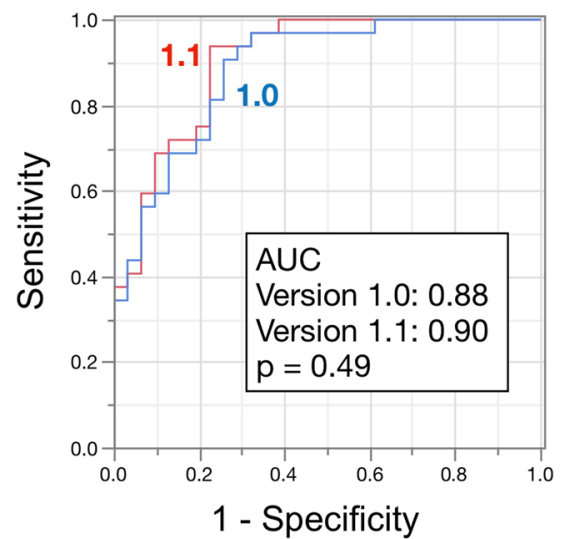

C Revascularization and/or OMI

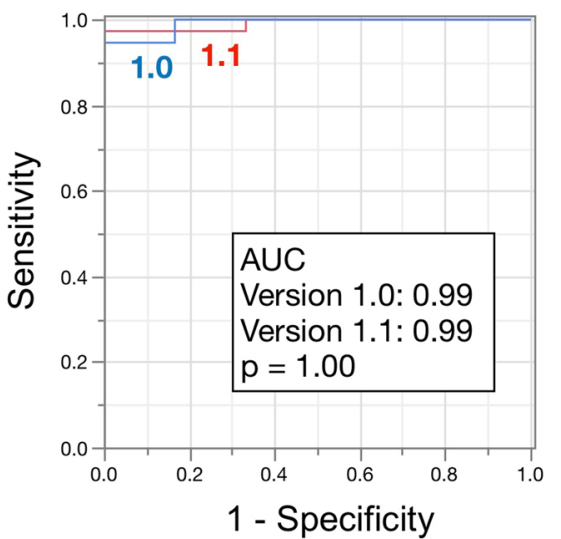

Fig. 2 Area under ROC curves to analyze stress defects in all patients (a). Without either revascularization or OMI $(n=47)(\mathbf{b})$. With revascularization and/or OMI (c) $(n=59)$. The statistical measures of sensitivity/specificity/accuracy were $83 / 86 / 84 \%$ and $94 / 81 / 90 \%$ for versions 1.0 and 1.1 , respectively, for all patients (a); $91 / 74 / 83 \%$ and

specificity for all patients were 94 and $81 \%$, respectively, with stress defect, and 87 and $96 \%$, respectively, with stress-rest difference.

Figure 4 shows the relationship between ANN probability and summed scores. The ANN probability steeply increased in the range of SSS 0 to 5 for both software versions. With respect to induced ischemia, although many points of data overlapped at an SDS of 0-1 and an ANN probability of 0 in version 1.0, ANN probability scattered in a low SDS range of $0-4$, indicating a higher prevalence of intermediate ANN probabilities in a range between 0.1 and 0.7 .
94/77/86\% for patients without either revascularization or OMI (b); and $95 / 100 / 95 \%$ and $97 / 100 / 98 \%$ for patients with revascularization and/or OMI (c). OMI old myocardial infarction, ROC receiver-operating characteristics

In addition to expert interpretation, diagnostic accuracy was assessed using coronary stenosis as another gold standard. Patients with revascularization and those with OMI were excluded from this analysis, and patients with either single-vessel disease (coronary stenosis $\geq 50 \%, n=22$ ) or no stenosis $(n=25)$ were included. The AUC for versions 1.0 and 1.1 were, respectively, 0.82 and $0.98(p=0.0099)$ when expert interpretation was the gold standard (Fig. 5a), and 0.66 and 0.81 , respectively $(p=0.0060)$, when coronary stenosis was the gold standard. These findings indicate that the diagnostic accuracy of version 1.1 had improved. The statistical measures of sensitivity/specificity were $93 / 94 \%$ 


\section{A All patients}

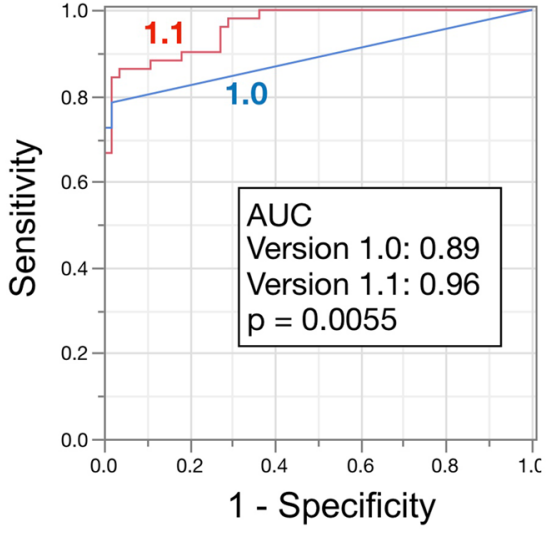

B Neither revascularization nor OMl

C Revascularization and/or OMI

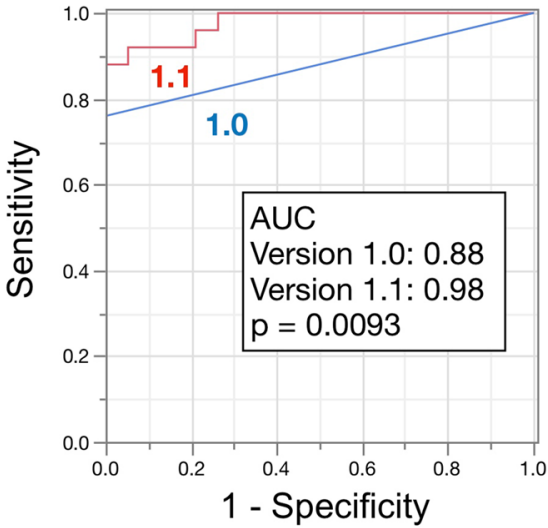

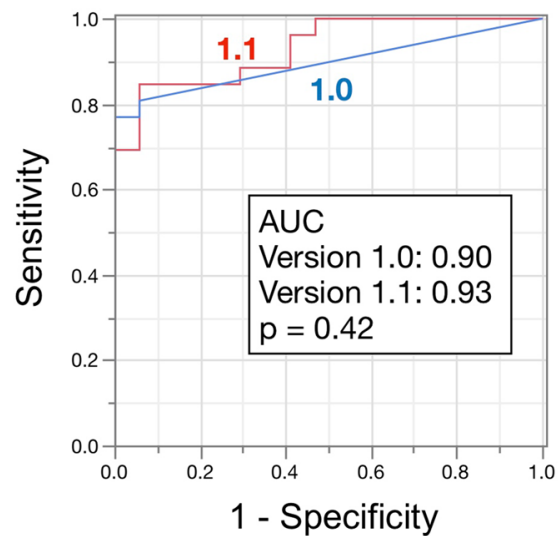

Fig. 3 Area under ROC curves to analyze induced ischemia. All patients (a), without either revascularization or OMI $(n=47)(\mathbf{b})$; with revascularization and/or OMI $(n=59)(\mathbf{c})$. The statistical measures of sensitivity/specificity/accuracy were 78/98/89\% and $87 / 96 / 92 \%$ for versions 1.0 and 1.1 , respectively, for all patients (a);
$76 / 100 / 90 \%$ and $88 / 100 / 95 \%$ for patients without either revascularization or OMI (b); and $77 / 100 / 86 \%$ and $85 / 94 / 88 \%$ for patients with revascularization and/or OMI (c). OMI old myocardial infarction, $R O C$ receiver-operating characteristics
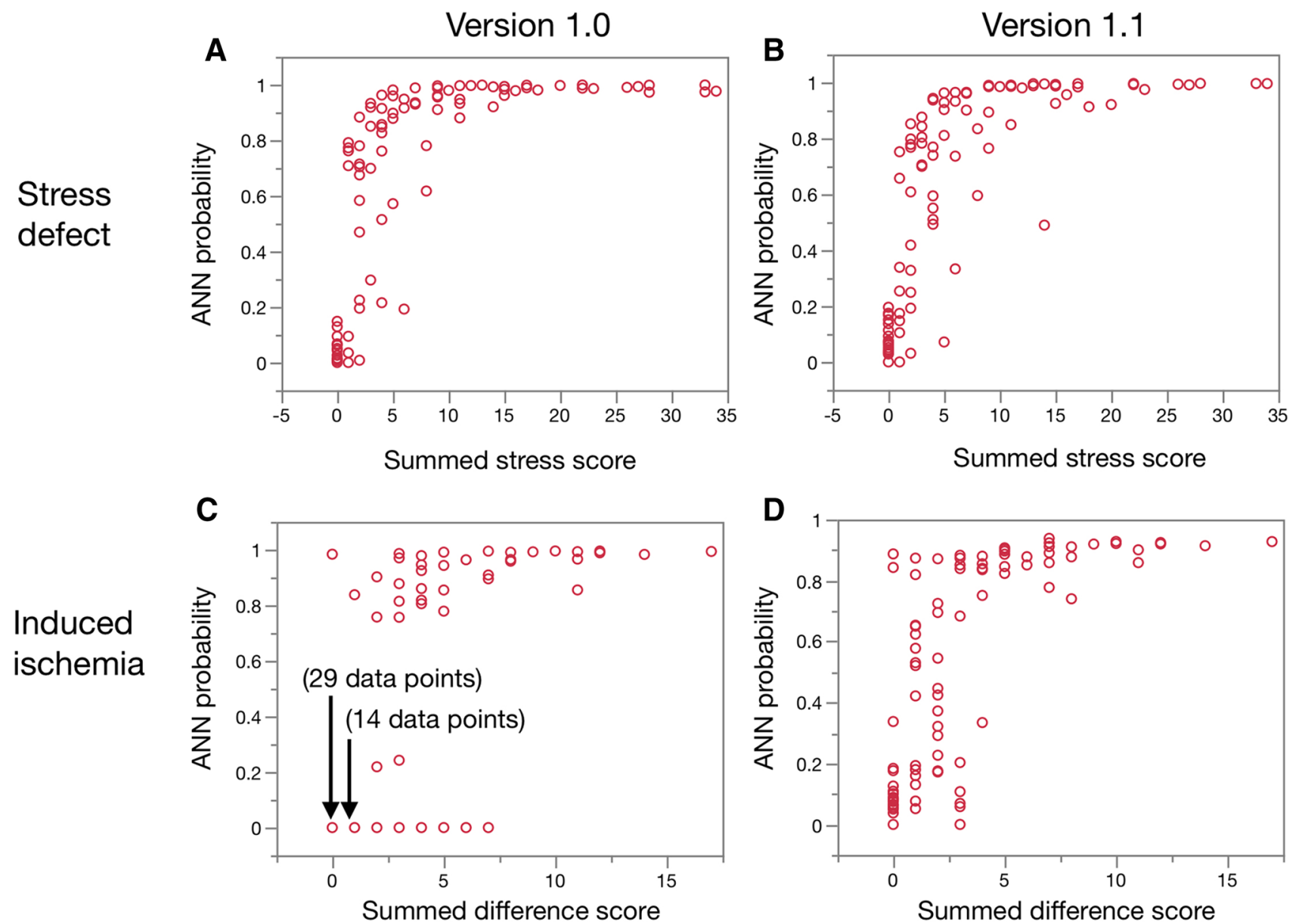

Fig. 4 Relationships between ANN probability and summed stress and summed difference scores between two software versions. Summed stress $(\mathbf{a}, \mathbf{b})$ and summed difference $(\mathbf{c}, \mathbf{d})$ scores for versions 1.0 and 1.1, respectively 
Fig. 5 ROC curves in subset of patients with either no stenosis or single-vessel disease. Patients with revascularization and/ or old myocardial infarction were excluded, and remaining 47 patients were analyzed. Gold standards were expert interpretation (a) and coronary stenosis (b). The statistical measures of sensitivity/specificity/accuracy were 64/100/89\% and $93 / 94 / 94 \%$ for versions 1.0 and 1.1 , respectively, with the gold standard of experts (a) and $37 / 96 / 68 \%$ and $64 / 92 / 79 \%$, respectively, with that of coronary stenosis (b)
A Gold standard: Experts

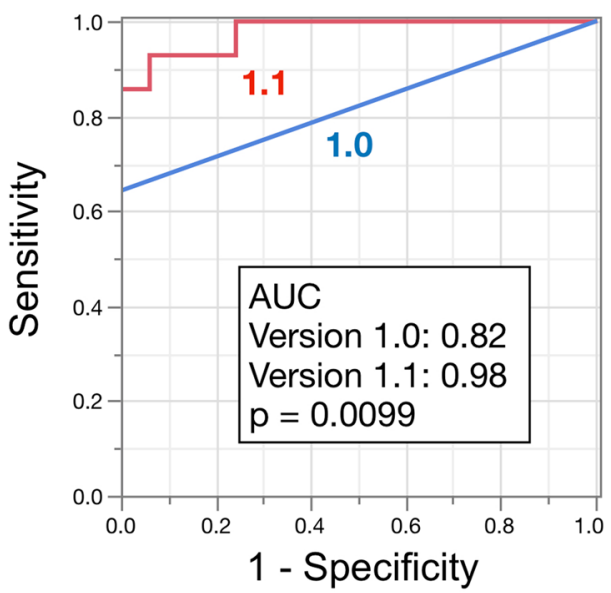

B Gold standard: Stenosis

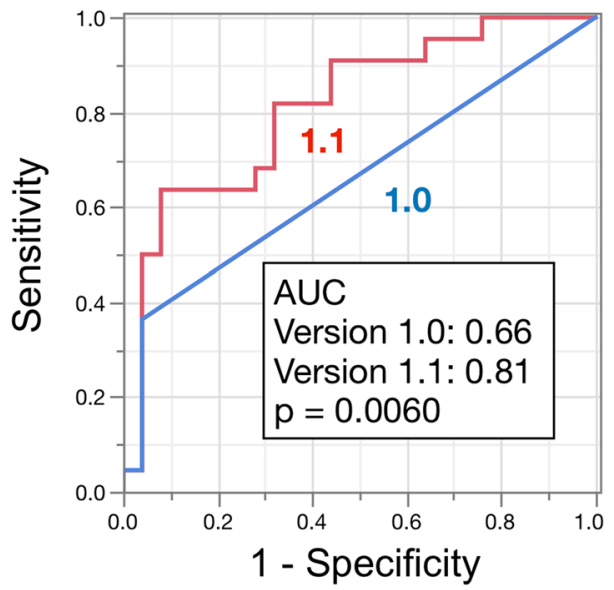

and $64 / 92 \%$ using the expert interpretation and coronary stenosis as the gold standard, respectively.

\section{Discussion}

The present study showed that the diagnostic accuracy of version 1.1 was better than that of version 1.0 when assessed using the same validation database. The diagnostic accuracy was obviously improved in patients without a history of myocardial infarction or coronary revascularization (that is, without modification by therapeutic intervention).

\section{Computer-aided diagnosis}

Visual assessment of myocardial perfusion SPECT for defects and reversibility is the initial step towards an appropriate diagnosis. Computer-assisted quantitation and evaluation play important roles in aiding visual assessment [11], and the most popular method of predicting prognosis has been defect scoring, such as SSS, SRS, and SDS using a 17or 20-segment model $[12,13]$. The amount of infarction and ischemia can also be determined by statistical analysis of the regional count distribution with assistance from normal databases fitted to a study population $[14,15]$. In contrast, the ANN determined the probability of abnormalities in candidate regions based on a learning experience similar to that used to train humans, which might be related to integrated information about defect size, location, extent, severity, regional wall motion, sex, and other factors. Therefore, the ANN might mimic the learning processes through which trainees develop the diagnostic ability to become nuclear cardiology experts. The superior diagnostic accuracy of the ANN system over scoring methods has already been established $[2,3]$.

\section{Gold standard for training}

The definition of a true diagnosis was based on the expert reading for both versions 1.0 and 1.1 in the present study. Since the target of the artificial intelligence applied in this study was to achieve diagnostic accuracy comparable to that of human experts, gold standards of coronary stenosis and fractional flow reserve were not applied. A gold standard comprising physicians' readings had been implemented in a study using the PERFEX system [1]. Although the detection of (for example) anatomical stenosis might be another target of ANN training, stenosis and physiological ischemia might not be identical [16]. Therefore, if experts cannot identify abnormalities on MPI acquired from patients with triple-vessel disease, an ANN would also be unable to do so. However, even when expert interpretation is defined as truth, the improved diagnostic ability of version 1.1 represents progress and support for clinical applications associated with coronary artery disease. Nevertheless, the ability of version 1.1 to accurately diagnose single-vessel disease was improved when coronary stenosis was the gold standard.

\section{Improvement for detecting ischemia}

The major improvement in version 1.1 was in its ability to detect stress-induced ischemia in patients without therapeutic modifications resulting from coronary intervention and without myocardial infarction. From our experience with applying ANN version 1.0, we found that small areas or slight degrees of ischemia were overlooked [3]. Therefore, during the development of the new version, we tried to select more candidate regions of abnormalities, and trained the ANN to identify minor degrees of abnormality. That is, the ANN learned to judge minor abnormalities as positive during the present training and development. The ANN was 
trained using supervised learning; the quality of the content that experts use to teach the ANN is an important part of software development using artificial intelligence.

Although we could not differentiate contribution of each feature in the neural network system, the integrated learning process was effective for improved diagnostic accuracy. Interestingly, intermediate ANN probability values were more often calculated for detecting ischemia by the version 1.1. Due to this change, sensitivity was improved for detecting ischemia while specificity was kept high (or low falsepositive rate).

\section{Neural network for clinical practice}

The practical method of applying the ANN to clinical practice should be considered. The relationship between ANN probability and defect scores is not linear [3, 4]. Summed stress, rest and difference scores all steeply increased when the ANN probability was $>0.80$, which means that the ANN probability could play a unique role in the diagnosis of coronary artery disease. Clinical decisions as to whether or not infarction and ischemia actually exist on MPI are often borderline, and the truth is not always clear. Under such circumstances, expressing perfusion abnormalities as probabilities might be more practical than simply announcing, for example, that ischemia is suspected or cannot be denied. However, diagnostic relevance should be further investigated since such approaches are not common to medical diagnostics. Since estimated areas of ischemia vary widely among physicians, the presence of defects and ischemia suggested by appropriate software packages would help to reduce the inter-observer variability of clinical interpretations [17].

\section{Limitations}

One limitation of the present study is that it included only 106 patients who had undergone coronary angiography. Considering that the diagnostic accuracy of version 1.1 has already been established based on 364 patients [4], the present study seems sufficiently valid for comparisons between the two versions. When patients with old myocardial infarction and post-revascularization conditions were included, truth could not be established. However, more precise analyses including follow-up and prognostic investigations might be feasible in future studies that include a sufficient number of patients.

\section{Conclusions}

The ANN version 1.0 was retrained with a Japanese database to create version 1.1 and then compared with the original ANN version 1.0 using the same dataset. The diagnostic ability of version 1.1 was better, mainly when patients had induced ischemia without revascularization and no myocardial infarction. The new ANN version 1.1 could serve in clinical practice as a second opinion for diagnoses based on stress myocardial perfusion images.

Acknowledgements The authors appreciate Kunihiko Yokoyama MD (Public Central Hospital of Matto Ishikawa, Hakusan, Japan) and Hisashi Bunko, MD (Kanazawa Cardiovascular Hospital, Kanazawa, Japan) for preparing the validation data. The authors are also grateful to Reo Usami for help with data analysis and Norma Foster for editorial assistance during manuscript preparation.

\section{Compliance with ethical standards}

Conflict of interest $\mathrm{KN}$ and $\mathrm{KO}$ have collaborated in research studies with FUJIFILM RI Pharma Co. Ltd. (Tokyo, Japan). Karin Toth and Lars Edenbrandt are full- and part-time employees, respectively, of EXINI Diagnostics, Lund, Sweden.

Funding This study was partly funded by JSPS Grants-in-Aid for Scientific Research (C) in Japan (PI: K. Nakajima, no. 15 K09947) and by FUJIFILM RI Pharma Co. Ltd. (Tokyo, Japan).

Open Access This article is distributed under the terms of the Creative Commons Attribution 4.0 International License (http://creativeco mmons.org/licenses/by/4.0/), which permits unrestricted use, distribution, and reproduction in any medium, provided you give appropriate credit to the original author(s) and the source, provide a link to the Creative Commons license, and indicate if changes were made.

\section{References}

1. Garcia EV, Cooke CD, Folks RD, Santana CA, Krawczynska EG, De Braal L, et al. Diagnostic performance of an expert system for the interpretation of myocardial perfusion SPECT studies. J Nucl Med. 2001;42:1185-1191.

2. Johansson L, Edenbrandt L, Nakajima K, Lomsky M, Svensson SE, Tragardh E. Computer-aided diagnosis system outperforms scoring analysis in myocardial perfusion imaging. J Nucl Cardiol. 2014;21:416-423.

3. Nakajima K, Matsuo S, Wakabayashi H, Yokoyama K, Bunko $\mathrm{H}$, Okuda K, et al. Diagnostic performance of artificial neural network for detecting ischemia in myocardial perfusion imaging. Circ J. 2015;79:1549-1556.

4. Nakajima K, Kudo T, Nakata T, Kiso K, Kasai T, Taniguchi Y, et al. Diagnostic accuracy of an artificial neural network compared with statistical quantitation of myocardial perfusion images: a Japanese multicenter study. Eur J Nucl Med Mol Imaging. 2017;44:2280-2289.

5. Henzlova MJ, Duvall WL, Einstein AJ, Travin MI, Verberne HJ. ASNC imaging guidelines for SPECT nuclear cardiology procedures: stress, protocols, and tracers. J Nucl Cardiol. 2016;23:606-639.

6. Nakajima K, Okuda K, Nystrom K, Richter J, Minarik D, Wakabayashi $\mathrm{H}$, et al. Improved quantification of small hearts for gated myocardial perfusion imaging. Eur J Nucl Med Mol Imaging. 2013;40:1163-1170.

7. Kondo C, Watanabe E, Momose M, Fukushima K, Abe K, Hagiwara $\mathrm{N}$, et al. In vivo validation of gated myocardial SPECT 
imaging for quantification of small hearts: comparison with cardiac MRI. EJNMMI Res. 2016;6:9.

8. Cerqueira MD, Weissman NJ, Dilsizian V, Jacobs AK, Kaul S, Laskey WK, et al. Standardized myocardial segmentation and nomenclature for tomographic imaging of the heart: a statement for healthcare professionals from the Cardiac Imaging Committee of the Council on Clinical Cardiology of the American Heart Association. Circulation. 2002;105:539-542.

9. Nakajima K. Normal values for nuclear cardiology: Japanese databases for myocardial perfusion, fatty acid and sympathetic imaging and left ventricular function. Ann Nucl Med. 2010;24:125-135.

10. Nakajima K, Matsumoto N, Kasai T, Matsuo S, Kiso K, Okuda $\mathrm{K}$. Normal values and standardization of parameters in nuclear cardiology: Japanese Society of Nuclear Medicine working group database. Ann Nucl Med. 2016;30:188-199.

11. Garcia EV, Klein JL, Taylor AT. Clinical decision support systems in myocardial perfusion imaging. J Nucl Cardiol. 2014;21:427 39; quiz 40.

12. Berman DS, Abidov A, Kang X, Hayes SW, Friedman JD, Sciammarella MG, et al. Prognostic validation of a 17-segment score derived from a 20 -segment score for myocardial perfusion SPECT interpretation. J Nucl Cardiol. 2004;11:414-423.
13. Germano G, Kavanagh PB, Slomka PJ, Van Kriekinge SD, Pollard G, Berman DS. Quantitation in gated perfusion SPECT imaging: the Cedars-Sinai approach. J Nucl Cardiol. 2007;14:433-454.

14. Slomka PJ, Nishina H, Berman DS, Akincioglu C, Abidov A, Friedman JD, et al. Automated quantification of myocardial perfusion SPECT using simplified normal limits. J Nucl Cardiol. 2005; 12:66-77.

15. Nakajima K, Okuda K, Kawano M, Matsuo S, Slomka P, Germano $\mathrm{G}$, et al. The importance of population-specific normal database for quantification of myocardial ischemia: comparison between Japanese 360 and 180-degree databases and a US database. J Nucl Cardiol. 2009;16:422-430.

16. Gupta A, Taqueti VR, van de Hoef TP, Bajaj NS, Bravo PE, Murthy VL, et al. Integrated noninvasive physiological assessment of coronary circulatory function and impact on cardiovascular mortality in patients with stable coronary artery disease. Circulation. 2017;136:2325-2336.

17. Edenbrandt L, Hoglund P, Frantz S, Hasbak P, Johansen A, Johansson L, et al. Area of ischemia assessed by physicians and software packages from myocardial perfusion scintigrams. BMC Med Imaging. 2014;14:5. 